\title{
Massera Type Theorems for Abstract Functional Differential Equations
}

\author{
By \\ Qing Liu ${ }^{1}$, Nguyen Van Minh ${ }^{2}$, G. Nguerekata ${ }^{3}$ and Rong Yuan ${ }^{4}$ \\ (Beijing Normal University, P.R. China ${ }^{1,4}$, University of West Georgia, USA ${ }^{2}$, \\ Morgan State University, USA $^{3}$ )
}

\begin{abstract}
The paper is concerned with conditions for the existence of almost periodic solutions of the following abstract functional differential equation $\dot{u}(t)=A u(t)+$ $[\mathscr{B} u](t)+f(t)$, where $A$ is a closed operator in a Banach space $\boldsymbol{X}, \mathscr{B}$ is a general bounded linear operator in the function space of all $X$-valued bounded and uniformly continuous functions that satisfies a so-called autonomous condition. We develop a general procedure to carry out the decomposition that does not need the wellposedness of the equations. The obtained conditions are of Massera type, which are stated in terms of spectral conditions of the operator $\mathscr{A}+\mathscr{B}$ and the spectrum of $f$. Moreover, we give conditions for the equation not to have quasi-periodic solutions with different structures of spectrum. The obtained results extend previous ones.

Key Words and Phrases. Almost periodic solution, Abstract functional differential equation, Massera type theorem, Quasi-periodic solution, Non-existence.

2000 Mathematics Subject Classification Numbers. 47D06; 34C27.
\end{abstract}

\section{Introduction}

In this paper we are concerned with the existence of almost periodic solutions to abstract functional differential equations of the form

$$
\dot{u}(t)=A u(t)+[\mathscr{B} u](t)+f(t),
$$

where $A$ is a closed operator on a Banach space $\boldsymbol{X}, \mathscr{B}$ is a bounded linear autonomous operator on $B U C(\boldsymbol{R}, \boldsymbol{X}), f$ is a $\boldsymbol{X}$-valued almost periodic function.

As is well known, in [18] Massera studied the following linear ordinary differential equation

$$
\dot{u}(t)=A(t) u(t)+f(t),
$$

where $A$ and $f$ are continuous, periodic with the same period $\tau$, and proved a classical theorem that is often referred to as Massera Theorem, saying that Eq. (1.2) has a periodic solution with period $\tau$ if and only if it has a bounded solution on the positive half line. The Massera Theorem has been extended to various kinds of differential equations. We refer the reader to $[4,15,16,27,3$, 11] and the references therein for more information in this direction. The 
method employed in these works is to prove the existence of periodic solutions via the existence of fixed points of the associated period maps. This method is no longer valid for more general problems dealing with almost periodic solutions, as one has no period map associated with a non-periodic equation. A new idea to study Massera type theorems for almost periodic solutions was developed in [23] that is referred to as spectral decomposition of bounded solutions. This idea can be carried out via the concept of evolution semigroups. Extensions of the results in [23] to various kind of equations were obtained in $[8,19,21,7]$. Note that the methods used in these papers are more or less based on the well-posedness of the equations, so they are no longer valid for the general setting of this paper in which the operator $A$ may not generate a semigroup, and especially, $\mathscr{B}$ is a very general functional operator.

In this paper we will develop a transparent operator theoretical framework for decomposition of bounded solutions of Eq. (1.1) with very general assumptions on $A$ and $\mathscr{B}$. This framework does not require any conditions for the well-posedness, for the variation-of-constants formula, so it can be applied to many kind of functional equations. We note that most of periodic functional evolution equations considered in previous works (see e.g. [8, 21, 27]) on Massera type theorems can be reduced to equations with constant coefficients by the partial Floquet representation developed in [10]. Therefore, our results obtained in this paper can be extended to these equations.

We now briefly outline our paper. In the next section we review some concepts such as almost periodicity, spectrum of a bounded function, and especially, the Loomis Theorem in the infinite dimensional case that is a key for our results. The section that follows contains the main results of this paper with two Massera type theorems (Theorems 12, 21) whose refinements are Corollary 28. A result on the non-existence of quasi-periodic solutions is Theorem 29. Finally, we give an application to show that our results can be applied to functional evolution equations of mixed type which are, in general, not well-posed, that is, the associated Cauchy Problem may have no solutions.

\section{Preliminaries}

In this section, we first give some notations and definitions. After recalling the concept of almost periodic functions we present the spectral theory of functions and several important properties which we need in the next sections. Finally, two lemmas on decomposition are proved.

\subsection{Notations and definitions}

Throughout this paper we will use the following notations: $N, \boldsymbol{Z}, \boldsymbol{Q}, \boldsymbol{R}, \boldsymbol{C}$ stand for the sets of natural, integer, rational, real and complex numbers, 
respectively. For any complex number $\lambda$, the notation $\operatorname{Re} \lambda$ stands for its real part. We always denote by $\boldsymbol{X}$ a given complex Banach space, and $B C(\boldsymbol{R}, \boldsymbol{X})$, $B U C(\boldsymbol{R}, \boldsymbol{X}), A P(\boldsymbol{X})$ the spaces of all $\boldsymbol{X}$-valued bounded continuous, bounded uniformly continuous and almost periodic functions on $\boldsymbol{R}$ with sup-norm, respectively. The translation group on $B C(\boldsymbol{R}, \boldsymbol{X})$ is denoted by $(S(t))_{t \in \boldsymbol{R}}$ and its generator $\mathscr{D}=d / d t$. The spaces $B C(\boldsymbol{R}, \boldsymbol{X}), B U C(\boldsymbol{R}, \boldsymbol{X}), A P(\boldsymbol{X})$ are translation-invariant. Given two complex Banach spaces $\boldsymbol{X}, \boldsymbol{Y}$, for any linear operator $T$ from $\boldsymbol{X}$ to $\boldsymbol{Y}$, as usual $\sigma(T), \rho(T), R(\lambda, T)=(\lambda-T)^{-1}$ are the notations of the spectrum, resolvent set, resolvent of $T$.

Let us denote by $\mathscr{A}$ the operator of multiplication by $A$ on $B U C(\boldsymbol{R}, \boldsymbol{X})$, that is, an operator with domain

$$
D(\mathscr{A}):=\{g \in B U C(\boldsymbol{R}, \boldsymbol{X}): g(t) \in D(A), \forall t \in \boldsymbol{R}, \text { and } A g(\cdot) \in B U C(\boldsymbol{R}, \boldsymbol{X})\}
$$

and $[\mathscr{A} u](t)=A u(t)$, for all $t \in \boldsymbol{R}$ and $u \in D(\mathscr{A})$.

Recall that two closed operators $A$ and $B$ on a Banach space $\boldsymbol{Y}$ with non-empty resolvent sets commute in the sense of resolvent commuting if $R(\lambda, A) R(\mu, B)=R(\mu, B) R(\lambda, A)$ for all $\lambda \in \rho(A), \mu \in \rho(B)$. Some elementary properties of commuting operators will be used in this paper whose proofs are left to the reader. For example, if $A$ generates a semigroup $(T(t))_{t \geq 0}$, then a closed operator $B$ commutes with $A$ if and only if it commutes with $T(t)$ for all $t \geq 0$.

Definition 1. A bounded linear operator $\mathscr{B}$ in $B U C(\boldsymbol{R}, \boldsymbol{X})$ is said to be autonomous if it commutes with the translation group $(S(t))_{t \in \boldsymbol{R}}$.

\subsection{Almost periodic functions}

Let $g$ be a continuous function on $\boldsymbol{R}$ taking values in a complex Banach space $\boldsymbol{X} . \quad g$ is said to be almost periodic in the sense of Bohr if to every $\varepsilon>0$, the set

$$
T(g, \varepsilon):=\left\{\tau: \sup _{t \in \boldsymbol{R}}\|g(t+\tau)-g(t)\| \leq \varepsilon\right\}
$$

is relatively dense in $\boldsymbol{R}$, i.e. there exists a number $l>0$ such that

$$
T(g, \varepsilon) \cap[t, t+l] \neq \varnothing, \quad \forall t \in \boldsymbol{R} .
$$

As is well known, for $g$ to be almost periodic it is necessary and sufficient that the family of functions $\{S(t) g\}_{t \in \boldsymbol{R}}$ is pre-compact in $B U C(\boldsymbol{R}, \boldsymbol{X})$.

For $g \in A P(X)$, let

$$
a(\lambda, g):=\lim _{T \rightarrow \infty} \frac{1}{2 T} \int_{-T}^{T} e^{-i \lambda t} g(t) d t, \quad \forall \lambda \in \boldsymbol{R} .
$$


It is known that $a(\lambda, g)$ is well defined and there are at most countably many points $\lambda$ such that $a(\lambda, g) \neq 0$. We call the set $\sigma_{b}(g):=\{\lambda: a(\lambda, g) \neq 0\}$ and $\mathfrak{M}:=\left\{\sum_{k=1}^{N} n_{k} \lambda_{k}: \lambda_{k} \in \sigma_{b}(g), n_{k} \in \boldsymbol{Z}, \forall N \in \boldsymbol{N}\right\}$ the Bohr spectrum and module of $g$, respectively.

Under the above notations, $g$ can be approximated uniformly on $\boldsymbol{R}$ by a sequence of trigonometric polynomial (see [14, Chap. 2])

$$
P_{n}(t):=\sum_{k=1}^{N(n)} a_{n, k} e^{i \lambda_{n, k} t}, \quad n=1,2, \ldots ; \lambda_{n, k} \in \sigma_{b}(g), t \in \boldsymbol{R} .
$$

And of course every function which can be approximated uniformly by a sequence of trigonometric polynomial is almost periodic.

A finite or countable set of real numbers $\Omega:=\left\{\omega_{1}, \omega_{2}, \ldots, \omega_{k}, \ldots\right\}$ is said to be rationally independent if for any $n \in N$, the following relation holds

$$
\left.\begin{array}{r}
r_{1} \omega_{1}+r_{2} \omega_{2}+\cdots+r_{n} \omega_{n}=0 \\
r_{1}, r_{2}, \ldots, r_{n} \in \boldsymbol{Q}
\end{array}\right\} \Rightarrow r_{1}=r_{2}=\cdots=r_{n}=0 .
$$

We call $\Omega$ a rational basis of $\sigma_{b}(g)$ if it is rationally independent and every $\lambda_{j} \in \sigma_{b}(g)$ is representable as a finite linear combination of the $\omega_{k}$ with rational coefficients, that is,

$$
\lambda_{j}=r_{1}^{(j)} \omega_{1}+r_{2}^{(j)} \omega_{2}+\cdots+r_{n}^{(j)} \omega_{n}, \quad r_{1}^{(j)}, r_{2}^{(j)}, \ldots, r_{n_{j}}^{(j)} \in \boldsymbol{Q}, \quad(j=1,2, \ldots) .
$$

Moreover, if all the $r_{k}^{(j)} \in \boldsymbol{Z}$, then the basis is called integer basis.

Definition 2. If $\mathfrak{M}(g)$ has a finite integer basis $\left\{\omega_{1}, \omega_{2}, \ldots, \omega_{k}\right\}$, then $g$ is called a quasi-periodic function with frequencies $\left(\omega_{1} / 2 \pi, \omega_{2} / 2 \pi, \ldots, \omega_{k} / 2 \pi\right)$. In the sequel, we also call $g k$-basic-frequency-quasi-periodic function, or $k$-quasiperiodic function.

From the definition it follows that if $g$ is $k$-quasi-periodic, it cannot be $l$-quasi-periodic for any $l<k$.

\subsection{Spectral theory of functions}

Let $g \in B C(\boldsymbol{R}, \boldsymbol{X})$, and let $\lambda \in \boldsymbol{C}$ such that $\operatorname{Re} \lambda \neq 0$. Then, obviously, the equation $\dot{x}=\lambda x, x \in \boldsymbol{R}$ has an exponential dichotomy. By the Perron Theorem in ODE, its non-homogeneous equation $\dot{x}=\lambda x+g(t)$ has a unique solution $x_{g, \lambda} \in B U C(\boldsymbol{R}, \boldsymbol{X})$. Moreover, $\quad x_{g, \lambda}=(\mathscr{D}-\lambda)^{-1} g$ for every $g \in B C(\boldsymbol{R}, \boldsymbol{X})$. Therefore, $\mathscr{D}$ is a closed operator on $B C(\boldsymbol{R}, \boldsymbol{X})$ and $\rho(\mathscr{D}) \supset \boldsymbol{C} \backslash i \boldsymbol{R}$, and $(\lambda-\mathscr{D})^{-1} g$, as a function of $\lambda$, is analytic everywhere in $\boldsymbol{C} \backslash i \boldsymbol{R}$.

Definition 3. The set of all reals $\xi \in \boldsymbol{R}$ such that the complex function $(\lambda-\mathscr{D})^{-1} g$ has no analytic extension to any neighborhood of $i \xi$ is said to be the uniform spectrum of $g$, and is denoted by $s p_{u}(g)$. 
Using the Green function to determine bounded solutions in the theory of ODE we have

$$
\begin{aligned}
(\lambda-\mathscr{D})^{-1} g(\xi) & =-x_{g, \lambda}(\xi) \\
& = \begin{cases}\int_{\xi}^{\infty} e^{\lambda(\xi-t)} g(t) d t, & \text { (if } \operatorname{Re} \lambda>0), \\
-\int_{-\infty}^{\xi} e^{\lambda(\xi-t)} g(t) d t, & \text { (if } \operatorname{Re} \lambda<0)\end{cases} \\
& = \begin{cases}\int_{0}^{\infty} e^{-\lambda \eta} g(\xi+\eta) d \eta, & (\text { if } \operatorname{Re} \lambda>0), \\
-\int_{-\infty}^{0} e^{-\lambda \eta} g(\xi+\eta) d \eta, & (\text { if } \operatorname{Re} \lambda<0) .\end{cases}
\end{aligned}
$$

By definition,

$$
\hat{g}(\lambda):=(\lambda-\mathscr{D})^{-1} g(0)= \begin{cases}\int_{0}^{\infty} e^{-\lambda \eta} g(\eta) d \eta, & \text { (if } \operatorname{Re} \lambda>0) \\ -\int_{-\infty}^{0} e^{-\lambda \eta} g(\eta) d \eta, & (\text { if } \operatorname{Re} \lambda<0)\end{cases}
$$

is called the Carleman-Laplace transform of $g$. Obviously, the CarlemanLaplace transform of $g$ is analytic in $\lambda \in \boldsymbol{C} \backslash i \boldsymbol{R}$.

Definition 4. Let $g \in B C(\boldsymbol{R}, \boldsymbol{X})$. The set of all reals $\xi$ such that $\hat{g}(\lambda)$ has no analytic extension to any neighborhood of $i \xi$ is called the Carleman spectrum of $g$ and is denoted by $\operatorname{sp}_{c}(g)$.

It is easy to see from the definitions that $s p_{c}(g) \subset s p_{u}(g)$. In [17], it is proved that they are actually coincide. As is well known (see e.g. [26]), the Carleman spectrum $s p_{c}(g)$ coincides with the Beurling spectrum $s p_{b}(g)$ defined as

$$
\operatorname{sp}_{b}(g):=\left\{\xi \in \boldsymbol{R}: \forall \varepsilon>0, \exists \varphi \in L^{1}(\boldsymbol{R}), \operatorname{supp} \tilde{\varphi} \subset(\xi-\varepsilon, \xi+\varepsilon), \varphi * g \neq 0\right\},
$$

where $\tilde{\varphi}$ is the Fourier transform of $\varphi$, that is,

$$
\tilde{\varphi}(\eta)=\int_{-\infty}^{\infty} e^{-i \eta t} \varphi(t) d t, \quad \eta \in \boldsymbol{R} .
$$

For this reason, for each $g \in B C(\boldsymbol{R}, \boldsymbol{X})$ we will denote simply by $\operatorname{sp}(g)$ the spectrum of $g$ in any sense mentioned above.

Below we give some properties of the spectrum of functions which we will need in the sequel.

Proposition 5. Let $g, g_{n} \in B U C(\boldsymbol{R}, \boldsymbol{X}), n \in \boldsymbol{N}$, such that $g_{n} \rightarrow g$ as $n \rightarrow \infty$. Then

i) $\operatorname{sp}(g)$ is closed,

ii) $s p(g(\cdot+h))=s p(g)$,

iii) If $\alpha \in C \backslash\{0\}$, then $s p(\alpha g)=s p(g)$,

iv) If $s p\left(g_{n}\right) \subset \Lambda$ for all $n \in N$, then $\operatorname{sp}(g) \subset \bar{\Lambda}$, 
v) If $A$ is a closed operator, $g(t) \in D(A), \forall t \in \boldsymbol{R}$ and $\mathscr{A} g \in B U C(\boldsymbol{R}, \boldsymbol{X})$, then $\operatorname{sp}(\mathscr{A} g) \subset \operatorname{sp}(g)$.

As a consequence of this proposition, for a closed subset of $\boldsymbol{R}$, say, $\Lambda$, then the following

$$
\begin{aligned}
\Lambda_{B U C}(\boldsymbol{X}) & :=\{g \in B U C(\boldsymbol{R}, \boldsymbol{X}): s p(g) \subset \Lambda\}, \\
\Lambda_{A P}(\boldsymbol{X}) & :=\{g \in A P(\boldsymbol{X}): s p(g) \subset \Lambda\},
\end{aligned}
$$

are closed translation-invariant subspaces of $B C(\boldsymbol{R}, \boldsymbol{X})$. Considering the translation group $(S(t))_{t \in \boldsymbol{R}}$ on $B U C(\boldsymbol{R}, \boldsymbol{X})$, we give a frequently used properties of spectrum.

Lemma 6. Denote $\mathscr{D}_{\Lambda}$ the restriction of $\mathscr{D}$ on $\Lambda_{B U C}(\boldsymbol{X})$. Then

$$
\sigma\left(\mathscr{D}_{\Lambda}\right)=i \Lambda \text {. }
$$

Proof. See [20, Lemma 3.3].

We also recall that the reduced spectrum $\operatorname{sp}_{A P}(g)$ is defined as follows:

$$
s p_{A P}(g):=\left\{\xi \in \boldsymbol{R}: \forall \varepsilon>0, \exists \varphi \in L^{1}(\boldsymbol{R}), \operatorname{supp} \tilde{\varphi} \subset(\xi-\varepsilon, \xi+\varepsilon), \varphi * f \notin A P(\boldsymbol{X})\right\} .
$$

Let us consider the quotient space $B U C(\boldsymbol{R}, \boldsymbol{X}) / A P(\boldsymbol{X})$. Since $(S(t))_{t \in \boldsymbol{R}}$ leaves $A P(\boldsymbol{X})$ invariant there is an induced translation group $(\tilde{\boldsymbol{S}}(t))_{t \in \boldsymbol{R}}$ on $B U C(\boldsymbol{R}, \boldsymbol{X}) / A P(\boldsymbol{X}) . \quad(\tilde{\boldsymbol{S}}(t))_{t \in \boldsymbol{R}}$ and its generator $\tilde{\mathscr{D}}$ are given by

$$
\begin{aligned}
\tilde{S}(t) \pi(g) & =\pi(S(t) g), & & t \in \boldsymbol{R}, g \in B U C(\boldsymbol{R}, \boldsymbol{X}), \\
\tilde{\mathscr{D}} \pi(g) & =\pi(\mathscr{D} g), & & g \in D(\mathscr{D}),
\end{aligned}
$$

where $\pi: B U C(\boldsymbol{R}, \boldsymbol{X}) \rightarrow B U C(\boldsymbol{R}, \boldsymbol{X}) / A P(\boldsymbol{X})$ denotes the quotient mapping. For $g \in B U C(\boldsymbol{R}, \boldsymbol{X})$, as in [1, the proof of Theorem 4.3, p. 374], $s p_{A P}(g)$ coincides with the set of all reals $\xi$ such that $(\lambda-\tilde{\mathscr{D}})^{-1} \pi(g)$ has no analytic extension to any neighborhood of $i \xi$. The following is the Loomis Theorem in the infinite dimensional case

Theorem 7. Let $\boldsymbol{X}$ be a Banach space that does not contain any subspaces isomorphic to $c_{0}$, and let $g$ be in $B U C(\boldsymbol{R}, \boldsymbol{X})$ with countable $s p_{A P}(g)$. Then, $g \in A P(\boldsymbol{X})$.

Proof. For the proof we refer the reader to $[1,2]$.

\subsection{Decomposition of function spaces}

In this subsection we will decompose a function space into direct sum of simpler ones in terms of spectrum. 
Lemma 8. Suppose the closed subset $\Lambda \subset \boldsymbol{R}$ can be represented as the disjoint union of a compact subsets $\Lambda^{1}$ and a closed set $\Lambda^{2}$, i.e.,

$$
\Lambda=\Lambda^{1} \cup \Lambda^{2} \text {. }
$$

Then,

$$
\Lambda_{B U C}(\boldsymbol{X})=\Lambda_{B U C}^{1}(\boldsymbol{X}) \oplus \Lambda_{B U C}^{2}(\boldsymbol{X}) .
$$

Moreover, the projections $P, Q$ corresponding to this splitting are determined from $R(\lambda, \mathscr{D})$ that commute with any operators that commute with $\mathscr{D}$ in the sense of resolvents commuting.

Proof. By Lemma 6, we have

$$
\sigma\left(\mathscr{D}_{\Lambda}\right)=i \Lambda=i \Lambda^{1} \cup i \Lambda^{2} .
$$

In case $\mathscr{D}_{\Lambda}$ is bounded we can prove the lemma by using the Riesz projection. For example, the subset $\Lambda$ is compact. Now, suppose $\mathscr{D}_{\Lambda}$ is unbounded. Then, for a fixed $\mu \in \rho\left(\mathscr{D}_{\Lambda}\right)$, we have $0 \in \sigma\left(R\left(\mu, \mathscr{D}_{\Lambda}\right)\right)$ and $\sigma\left(R\left(\mu, \mathscr{D}_{\Lambda}\right)\right) \backslash\{0\}=$ $\left(\mu-\sigma\left(\mathscr{D}_{\Lambda}\right)\right)^{-1}:=\left\{1 /(\mu-\lambda): \lambda \in \sigma\left(\mathscr{D}_{\Lambda}\right)\right\}$ (see [6, Chapter iv, sect. 1, p. 227]). Therefore, we obtain

$$
\begin{aligned}
\sigma\left(R\left(\mu, \mathscr{D}_{\Lambda}\right)\right) & =\left(\mu-\sigma\left(\mathscr{D}_{\Lambda^{1}}\right)\right)^{-1} \cup\left(\mu-\sigma\left(\mathscr{D}_{\Lambda^{2}}\right)\right)^{-1} \cup\{0\} \\
& =: \tau_{1} \cup \tau_{2},
\end{aligned}
$$

where $\tau_{1}:=\left(\mu-\sigma\left(\mathscr{D}_{\Lambda^{1}}\right)\right)^{-1}, \tau_{2}:=\left(\mu-\sigma\left(\mathscr{D}_{\Lambda^{2}}\right)\right)^{-1} \cup\{0\}$ are compact and disjoint subsets of $\boldsymbol{C}$.

Now let

$$
P:=\frac{1}{2 \pi i} \int_{\gamma} R\left(\lambda, R\left(\mu, \mathscr{D}_{\Lambda}\right)\right) d \lambda,
$$

where $\gamma$ is a Jordan path in the complement of $\tau_{2}$ and enclosing $\tau_{1}$. This projection commutes with any operators that commute with $\mathscr{D}_{\Lambda}$ in the sense of resolvents commuting and yields the spectral decomposition

$$
\Lambda_{B U C}(\boldsymbol{X})=\boldsymbol{X}_{1} \oplus \boldsymbol{X}_{2}
$$

with $\mathscr{D}_{\Lambda}$-invariant spaces $\boldsymbol{X}_{1}=P \Lambda_{B U C}(\boldsymbol{X}), X_{2}=Q \Lambda_{B U C}(\boldsymbol{X}), Q:=I-P$. The restrictions $\mathscr{D}_{\boldsymbol{X}_{1}}$ and $\mathscr{D}_{\boldsymbol{X}_{2}}$ of $\mathscr{D}_{\Lambda}$ satisfy

$$
\sigma\left(\mathscr{D}_{\boldsymbol{X}_{1}}\right)=i \Lambda^{1}, \quad \sigma\left(\mathscr{D}_{\boldsymbol{X}_{2}}\right)=i \Lambda^{2} .
$$

Let $\xi \in \boldsymbol{R} \backslash \Lambda^{1}$. Then, for every $g \in \boldsymbol{X}_{1}, \quad(\lambda-\mathscr{D})^{-1} g=\left(\lambda-\mathscr{D}_{\boldsymbol{X}_{1}}\right)^{-1} g$ has an analytic extension to some neighborhood of $i \xi$. So $\operatorname{sp}(g) \subset \Lambda^{1}$, and $\boldsymbol{X}_{1} \subset$ $\Lambda_{B U C}^{1}(\boldsymbol{X})$. Similarly, $\boldsymbol{X}_{2} \subset \Lambda_{B U C}^{2}(\boldsymbol{X})$. On the other hand, since $\Lambda_{B U C}^{1}(\boldsymbol{X}) \cap$ 
$\Lambda_{B U C}^{2}(\boldsymbol{X})=\{0\}$, we obtain

$$
\Lambda_{B U C}(\boldsymbol{X})=\Lambda_{B U C}^{1}(\boldsymbol{X}) \oplus \Lambda_{B U C}^{2}(\boldsymbol{X}) .
$$

When both sets $\Lambda^{1}$ and $\Lambda^{2}$ are not compact we have the following decomposition. Let $\Sigma, \Sigma_{1}, \Sigma_{2}$ be closed subsets of the unit circle $\Gamma$ such that $\Sigma$ is the disjoint union of $\Sigma_{1}$ and $\Sigma_{2}$. Denote

$$
\begin{gathered}
\Lambda=\left\{\xi \in \boldsymbol{R}: e^{i \xi} \in \Sigma\right\}, \\
\Lambda^{1}=\left\{\xi \in \boldsymbol{R}: e^{i \xi} \in \Sigma_{1}\right\}, \\
\Lambda^{2}=\left\{\xi \in \boldsymbol{R}: e^{i \xi} \in \Sigma_{2}\right\} .
\end{gathered}
$$

Lemma 9. Under the above notations, one has

$$
\Lambda_{B U C}(\boldsymbol{X})=\Lambda_{B U C}^{1}(\boldsymbol{X}) \oplus \Lambda_{B U C}^{2}(\boldsymbol{X}) .
$$

Moreover, the projections $P, Q$ corresponding to this splitting are determined as the Riesz spectral projections of the translation $S(1)$ on $\Lambda_{B U C}(\boldsymbol{X})$ with respect to $\Sigma_{1}$ and $\Sigma_{2}$, so each operator commuting with translation commutes with $P, Q$ as well.

Proof. Consider the operators $\mathscr{D}_{\Lambda}$ on $\Lambda_{B U C}(\boldsymbol{X}),\left(S_{\Lambda}(t)\right)_{t \in \boldsymbol{R}}$ on $\Lambda_{B U C}(\boldsymbol{X})$. By the Weak Spectral Mapping Theorem, we obtain

$$
\sigma\left(S_{\Lambda}(1)\right)=\overline{e^{\sigma\left(\mathscr{D}_{\Lambda_{B U C}(\boldsymbol{X})}\right)}}=\overline{e^{i \Lambda}}=\Sigma=\Sigma_{1} \cup \Sigma_{2} .
$$

Using the Riesz projection $P$ on $\Lambda_{B U C}(\boldsymbol{X})$ for $S(1)$, we have

$$
\Lambda_{B U C}(\boldsymbol{X})=P \Lambda_{B U C}(\boldsymbol{X}) \oplus Q \Lambda_{B U C}(\boldsymbol{X}),
$$

where $P:=(2 \pi i)^{-1} \int_{\gamma} R\left(\lambda, S_{\Lambda}(1)\right) d \lambda, \quad Q:=I-P, \quad \gamma$ is a simple contour in the complement of $\Sigma_{2}$ and enclosing $\Sigma_{1}$. We will prove that $P \Lambda_{B U C}(\boldsymbol{X})=$ $\Lambda_{B U C}^{1}(\boldsymbol{X})$. In fact, by the Weak Spectral Mapping Theorem

$$
\overline{e^{\sigma\left(\left.\mathscr{D}\right|_{P \Lambda_{B U C}(\boldsymbol{X})}\right)}}=\sigma\left(\left.S_{\Lambda}(1)\right|_{P \Lambda_{B U C}(\boldsymbol{X})}\right)=\Sigma_{1}=\overline{e^{i \Lambda^{1}}} .
$$

So

$$
\sigma\left(\left.\mathscr{D}\right|_{P \Lambda_{B U C}(\boldsymbol{X})}\right) \subset i \Lambda^{1} .
$$

Therefore, for each $u \in P \Lambda_{B U C}(\boldsymbol{X})$ one has $s p(u) \subset \Lambda^{1}$. Similarly, if $w \in$ $Q \Lambda_{B U C}(\boldsymbol{X})$, then $\operatorname{sp}(w) \subset \Lambda^{2}$. Obviously, $\Lambda_{B U C}^{1}(\boldsymbol{X}) \cap \Lambda_{B U C}^{2}(\boldsymbol{X})=\{0\}$ and they are closed subspaces of $\Lambda_{B U C}(\boldsymbol{X})$. So, we have

$$
\Lambda_{B U C}(\boldsymbol{X})=\Lambda_{B U C}^{1}(\boldsymbol{X}) \oplus \Lambda_{B U C}^{2}(\boldsymbol{X})
$$

and the corresponding projection $P=(2 \pi i)^{-1} \int_{\gamma} R\left(\lambda, S_{\Lambda}(1)\right) d \lambda$. Note that $P$ is 
determined from $S(t)$, so it commutes with any operators that commute with $S(t)$, or $\mathscr{D}$.

\section{Main results}

We begin this section by recalling some concept of solutions

Definition 10. A function $u \in B U C(\boldsymbol{R}, \boldsymbol{X})$ is said to be a classical solution of Eq. (1.1) if $\dot{u}(t)$ exists as an element of $B U C(\boldsymbol{R}, \boldsymbol{X})$ such that for all $t$, $u(t) \in D(A)$, and Eq. (1.1) holds.

Definition 11. A function $u \in B U C(\boldsymbol{R}, \boldsymbol{X})$ is said to be a mild solution of Eq. (1.1) if for all $t \in \boldsymbol{R}, \int_{0}^{t} u(\xi) d \xi \in D(A)$, and the following equation is satisfied:

$$
u(t)-u(0)=A \int_{0}^{t} u(\xi) d \xi+\int_{0}^{t}[\mathscr{B} u(\xi)+f(\xi)] d \xi .
$$

Obviously, every classical solution is a mild solution, but not vice versa in general. We can check that a mild solution $u$ that is continuously differentiable, and $u(t) \in D(A)$ for all $t$, is a classical one. We note that (see e.g. [2, 12, 20]) if $A$ generates a semigroup $(T(t))_{t \geq 0}$, then it is a mild solution in the sense that it satisfies the following integral equation

$$
u(t)=T(t-s) u(s)+\int_{s}^{t} T(t-\xi)[\mathscr{B} u(\xi)+f(\xi)] d \xi, \quad \forall t \geq s .
$$

In the sequel, if $A$ is an operator in a Banach space $\boldsymbol{Y}$, we use the notation

$$
\sigma_{i}(A):=\{\xi \in \boldsymbol{R}: i \xi \in \sigma(A)\} .
$$

Theorem 12. Let Eq. (1.1) have a mild solution $u$ in $B U C(\boldsymbol{R}, \boldsymbol{X})$, $\sigma_{i}(\mathscr{A}+\mathscr{B}) \backslash s p(f)$ be compact, $s p(f)$ be countable, and $\boldsymbol{X}$ do not contain any subspace isomorphic to $c_{0}$. Then Eq. (1.1) has an almost periodic mild solution $u_{f}$ with $s p\left(u_{f}\right) \subset s p(f)$.

We would like to present some useful results before giving the proof of Theorem 12.

Definition 13. Let $\Lambda$ be any closed subset of the real line. We define an operator $L_{\Lambda}$ as follows: $u \in D\left(L_{\Lambda}\right)$ if and only if $u \in \Lambda_{B U C}(\boldsymbol{X}), \int_{0}^{t} u(\xi) d \xi \in$ $D(A)$ for all $t$ and there is $g \in \Lambda_{B U C}(\boldsymbol{X})$ such that

$$
u(t)-u(0)=A \int_{0}^{t} u(\xi) d \xi+\int_{0}^{t} g(\xi) d \xi, \quad \forall t \in \boldsymbol{R},
$$

and in this case $L_{\Lambda} u:=g$. 
At this point, we can check easily that $L$ is a linear operator, but we do not know if this operator is a single-valued operator. The next lemma will clarify this. Note that since $\mathscr{B}$ is bounded and commutes with translations, one can easily show that it leaves $\Lambda_{B U C}(\boldsymbol{X})$ invariant. Therefore, by abuse of notation we will still use $\mathscr{B}$ to denote its restriction to $\Lambda_{B U C}(\boldsymbol{X})$ if this does not cause any danger of confusion.

Lemma 14. The operator $L_{\Lambda}$ is a linear single-valued and closed operator on $\Lambda_{B U C}(\boldsymbol{X})$, so $L_{\Lambda}-\mathscr{B}$ is a closed single-valued operator in $\Lambda_{B U C}(\boldsymbol{X})$.

Proof. First, we show that $L_{\Lambda}$ is a single-valued closed operator. Suppose $u \in D\left(L_{\Lambda}\right), g_{1}, g_{2} \in \Lambda_{B U C}(\boldsymbol{X})$ such that

$$
L_{\Lambda} u=g_{1}, \quad L_{\Lambda} u=g_{2},
$$

then

$$
\int_{0}^{t}\left(g_{1}(\xi)-g_{2}(\xi)\right) d \xi=0, \quad \forall t
$$

thus

$$
\frac{1}{t-s} \int_{0}^{t}\left(g_{1}(\xi)-g_{2}(\xi)\right) d \xi=0, \quad \forall t>s .
$$

Fixing $t$ and letting $s \rightarrow t$ we obtain $g_{1}(t)-g_{2}(t)=0$, i.e. $L_{\Lambda}$ is single-valued.

Suppose $u, u_{n} \in D\left(L_{\Lambda}\right), \quad \forall n \in \boldsymbol{N}, \quad g, g_{n} \in \Lambda_{B U C}(\boldsymbol{X}), \quad \forall n \in \boldsymbol{N}, \quad$ such that $L_{\Lambda} u_{n}=g_{n}$ and

$$
\lim _{n \rightarrow \infty} u_{n}=u, \quad \lim _{n \rightarrow \infty} g_{n}=g
$$

in the sup-norm topology of $B U C(\boldsymbol{R}, \boldsymbol{X})$, then

$$
\begin{array}{llll}
\int_{0}^{t} u_{n}(\xi) d \xi \rightarrow \int_{0}^{t} u(\xi) d \xi, & (n \rightarrow \infty) & \forall t, \\
\int_{0}^{t} g_{n}(\xi) d \xi \rightarrow \int_{0}^{t} g(\xi) d \xi, & (n \rightarrow \infty) & \forall t .
\end{array}
$$

By the closedness of $A$, for each fixed $t \in \boldsymbol{R}$ we have

$$
u(t)-u(0)=A \int_{0}^{t} u(\xi) d \xi+\int_{0}^{t} g(\xi) d \xi, \quad \forall t .
$$

This shows that $L_{\Lambda}$ is closed.

Finally, since $\mathscr{B}$ is bounded is bounded $L_{\Lambda}-\mathscr{B}$ is a closed operator on $\Lambda_{\text {BUC }}(\boldsymbol{X})$. 
For the relation among the spectrum of mild solutions, $\mathscr{A}+\mathscr{B}$ and $f$ we have the following theorem that has been known for abstract ODE (see e.g. [2, 14]).

Theorem 15. Let $u$ be a mild solution of Eq. (1.1) on $\boldsymbol{R}$. Then

$$
s p(u) \subset \sigma_{i}(\mathscr{A}+\mathscr{B}) \cup s p(f),
$$

where $\sigma_{i}(\mathscr{A}+\mathscr{B}):=\{\xi \in \boldsymbol{R}: i \xi \in \sigma(\mathscr{A}+\mathscr{B})\}$.

Proof. For $\operatorname{Re} \lambda>0$ taking the Carleman-Laplace transform of both sides of Eq. (3.1) we have

$$
\left.\hat{u}(\lambda)-\frac{1}{\lambda} u(0)=\frac{1}{\lambda} A \hat{u}(\lambda)+\frac{1}{\lambda} \widehat{[\mathscr{B} u}\right](\lambda)+\frac{1}{\lambda} \hat{f}(\lambda) .
$$

Therefore,

$$
\lambda \hat{u}(\lambda)-u(0)=A \hat{u}(\lambda)+\widehat{[\mathscr{B} u}(\lambda)+\hat{f}(\lambda) .
$$

Similarly, since $S(s) u$ is again a mild solution of Eq. (1.1) with $f$ replaced by $S(s) f$ for each fixed $s \in \boldsymbol{R}$, we have

$$
\lambda \widehat{S(s) u}(\lambda)-[S(s) u](0)=A \widehat{S(s) u}(\lambda)+[\mathscr{B S ( s )} u](\lambda)+\widehat{S(s) f}(\lambda) .
$$

By (2.1), this means

$$
\lambda R(\lambda, \mathscr{D}) u-u=\mathscr{A} R(\lambda, \mathscr{D}) u+R(\lambda, \mathscr{D}) \mathscr{B} u+R(\lambda, \mathscr{D}) f .
$$

Since $\mathscr{B}$ commutes with $\mathscr{D}$, we have

$$
\lambda R(\lambda, \mathscr{D}) u-u=\mathscr{A} R(\lambda, \mathscr{D}) u+\mathscr{B} R(\lambda, \mathscr{D}) u+R(\lambda, \mathscr{D}) f .
$$

Suppose $\xi$ is a real such that $\xi \notin s p(f)$, and $i \xi \notin \sigma(\mathscr{A}+\mathscr{B})$. Then $R(\lambda, \mathscr{D}) f$, and $[\lambda-\mathscr{A}-\mathscr{B}]^{-1}$ are extendable to analytic functions around $i \xi$. And for such a $\xi$ we have

$$
(\lambda-\mathscr{A}-\mathscr{B})^{-1}[R(\lambda, \mathscr{D}) f+u]=R(\lambda, \mathscr{D}) u .
$$

Therefore, for such a $\xi, R(\lambda, \mathscr{D}) u$ has an analytic extension to a neighborhood of $i \xi$. This show that $\xi \notin s p(u)$. The theorem is proved.

Lemma 14 and Theorem 15 yield the following

Lemma 16. Let $\Lambda=\sigma_{i}(\mathscr{A}+\mathscr{B}) \cup s p(f)$. Then a function $u \in B U C(\boldsymbol{R}, \boldsymbol{X})$ is a mild solution of Eq. (1.1) if and only if

$$
\left(L_{\Lambda}-\mathscr{B}\right) u=f .
$$

Proof. By Lemma 14 the operator $L_{\Lambda}$ is well-defined as a single-valued operator. Obviously, if $u$ is a solution of Eq. (3.7), then by the definition of the operator $L_{\Lambda}$ it satisfies Eq. (3.1), so it is a mild solution of Eq. (1.1). 
Conversely, let $u \in B U C(\boldsymbol{R}, \boldsymbol{X})$ be a mild solution of Eq. (1.1). Then, by Theorem 15 it is in $\Lambda_{B U C}(\boldsymbol{X})$, so, by the definition of the operator $L_{\Lambda}$, it satisfies Eq. (3.7).

Noticing that $f$ is almost periodic, similarly as the proof of Theorem 15, we can obtain the following theorem about the reduced spectrum of mild solutions.

Theorem 17. Let $u$ be a mild solution of Eq. (1.1) on $\boldsymbol{R}$. Then,

$$
s p_{A P}(u) \subset \sigma_{i}(\mathscr{A}+\mathscr{B}) .
$$

Proof. Recall that $\mathscr{A}+\mathscr{B}$ is an operator defined in $B U C(\boldsymbol{R}, \boldsymbol{X})$. By (3.6),

$$
[\lambda-\mathscr{A}-\mathscr{B}] R(\lambda, \mathscr{D}) u=R(\lambda, \mathscr{D}) f+u .
$$

Let $\xi \in \boldsymbol{R}$ such that $i \xi \in \rho(\mathscr{A}+\mathscr{B})$. Then, the resolvent $R(\lambda, \mathscr{A}+\mathscr{B})$ exists, and is analytic in a neighborhood of $i \xi$ in $\boldsymbol{C}$. Therefore, in a neighborhood of $i \xi$ if $\operatorname{Re} \lambda \neq 0$, by (3.9) we have

$$
R(\lambda, \mathscr{D}) u=R(\lambda, \mathscr{A}+\mathscr{B})(R(\lambda, \mathscr{D}) f+u) .
$$

Note that each bounded linear operator in $B U C(\boldsymbol{R}, \boldsymbol{X})$ that commutes with the translations must map $A P(X)$ into itself. Therefore, for $\operatorname{Re} \lambda \neq 0, R(\lambda, \mathscr{D})$ maps $A P(\boldsymbol{X})$ into itself. From the autonomousness of $\mathscr{B}$, it follows also that $R(\lambda, \mathscr{A}+\mathscr{B})$ commutes with the translations, so it leaves $A P(X)$ invariant. This means that the operators $R(\lambda, \mathscr{D})$ and $R(\lambda, \mathscr{A}+\mathscr{B})$ induce operators $R(\widetilde{\lambda, \mathscr{D}})=R(\lambda, \tilde{\mathscr{D}})$ and $R(\lambda, \widetilde{\mathscr{A}}+\mathscr{B})$ in $B U C(\boldsymbol{R}, \boldsymbol{X}) / A P(\boldsymbol{X})$, respectively. Since $f \in A P(\boldsymbol{R}, \boldsymbol{X})$, we have $R(\lambda, \tilde{\mathscr{D}}) \pi(f)=0$. And hence, by (3.10) we have

$$
R(\lambda, \tilde{\mathscr{D}}) \pi(u)=R(\lambda, \widetilde{\mathscr{A}}+\mathscr{B}) \pi(u) .
$$

Therefore, $R(\lambda, \widetilde{\mathscr{A}}+\mathscr{B}) \pi(u)$ is a natural analytic extension to a neighborhood of $i \xi$ for $R(\lambda, \tilde{D}) \pi(u)$. This shows that $\xi \notin s p_{A P}(u)$ and the proof is completed.

By Theorem 7 and Theorem 17 we obtain the following corollary immediately.

Corollary 18. Let $\sigma_{i}(\mathscr{A}+\mathscr{B})$ be countable. Then every bounded and uniformly continuous mild solution of Eq. (1.1) is almost periodic provided $\boldsymbol{X}$ does not contain any subspace isomorphic to $c_{0}$.

The following lemma is standard (see [13, Proposition III.6.5]).

Lemma 19. Let $A$ be a closed operator on a Banach space $\boldsymbol{X}$ such that $\rho(A) \neq \varnothing$, and let $B$ be a bounded operator on $\boldsymbol{X}$. Then, $A$ commutes with $B$ in 
the sense of resolvents commuting if and only if they commute in the sense that $B D(A) \subset D(A)$ and $A B x=B A x$ for all $x \in D(A)$.

Now we are ready to complete the proof of Theorem 12.

Proof of Theorem 12. Let $\Lambda^{1}:=\sigma_{i}(\mathscr{A}+\mathscr{B}) \backslash s p(f)$ and $\Lambda^{2}:=s p(f)$. By Theorem 15, $s p(u) \subset \Lambda:=\Lambda^{1} \cup \Lambda^{2}$. By the compactness of $\Lambda^{1}$ and Lemma 8 we have

$$
\Lambda_{B U C}(\boldsymbol{X})=\Lambda_{B U C}^{1}(\boldsymbol{X}) \oplus \Lambda_{B U C}^{2}(\boldsymbol{X}),
$$

and the projections $P, Q$ corresponding to this splitting commute with each operator that commutes with $\mathscr{D}_{A}$ in the sense of resolvents commuting. Note that $L_{\Lambda}$ commutes with all translations, so it commutes with $\mathscr{D}_{\Lambda}$. By Lemma 16 we have

$$
Q\left[L_{\Lambda}-\mathscr{B}\right] u=Q f=f .
$$

By Lemma 19, $Q\left[L_{\Lambda}-\mathscr{B}\right] u=\left[L_{\Lambda}-\mathscr{B}\right] Q u$, and letting $Q u=w$, we have

$$
\left[L_{\Lambda}-\mathscr{B}\right] w=f .
$$

That is, $w \in \Lambda_{B U C}^{2}(\boldsymbol{X})$ is a mild solution of Eq. (1.1). Since $\Lambda^{2}$ is countable and $\boldsymbol{X}$ does not contain any subspace isomorphic to $c_{0}$, by Theorem $7, w$ is almost periodic. The theorem is proved.

Now we consider the case when $\sigma_{i}(\mathscr{A}+\mathscr{B}) \backslash s p(f)$ may not be compact.

Theorem 20. Suppose that Eq. (1.1) has a mild solution $u \in B U C(\boldsymbol{R}, \boldsymbol{X})$, and $\overline{e^{i \sigma_{i}(\mathscr{A}+B)}} \backslash \overline{e^{i s p(f)}}$ is closed. Then, there exists a mild solution $w$ of Eq. (1.1) such that

$$
\text { Proof. Let } \quad \Sigma:=\overline{e^{i \sigma_{i}(\mathscr{A}+B)}} \cup \overline{e^{i s p(f)}}, \quad \Sigma_{1}:=\overline{e^{i \sigma_{i}(\mathscr{A}+B)}} \backslash \overline{e^{i s p(f)}}, \quad \Sigma_{2}:=\overline{e^{i s p(f)}} .
$$
Obviously, $\Sigma, \Sigma_{1}, \Sigma_{2}$ are closed. Moreover, by the assumption, $\Sigma_{1} \cap \Sigma_{2}=\varnothing$. Therefore, by Lemma 9 , if we define

$$
\begin{aligned}
\Lambda & =\left\{\xi \in \boldsymbol{R}: e^{i \xi} \in \Sigma\right\}, \\
\Lambda^{1} & =\left\{\xi \in \boldsymbol{R}: e^{i \xi} \in \Sigma_{1}\right\}, \\
\Lambda^{2} & =\left\{\xi \in \boldsymbol{R}: e^{i \xi} \in \Sigma_{2}\right\},
\end{aligned}
$$

then

$$
\Lambda_{B U C}(\boldsymbol{X})=\Lambda_{B U C}^{1}(\boldsymbol{X}) \oplus \Lambda_{B U C}^{2}(\boldsymbol{X}) .
$$

Moreover, the projections $P$ and $Q$ corresponding to this splitting commute with 
any operators that commute with translations. In the same way as in the proof of Theorem 12 we can show that $w:=Q u$ is the sought mild solution.

Now we prove a Massera type theorem for almost periodic solutions of Eq. (1.1) when $\sigma_{i}(\mathscr{A}+\mathscr{B}) \backslash s p(f)$ may not be compact.

Theorem 21. In addition to the conditions of Theorem 20 assume that $\boldsymbol{X}$ does not contain any subspace isomorphic to $c_{0}$ and $\overline{e^{i s p(f)}}$ is countable. Then Eq. (1.1) has an almost periodic mild solution $w$ such that

$$
e^{i s p(w)} \subset \overline{e^{i s p(f)}} .
$$

Proof. By Theorem 20, we obtain that $e^{i s p(w)}$ is countable. This implies that $s p(w)$ is countable. By Loomis' Theorem we have $w \in A P(\boldsymbol{X})$.

Let Eq. (1.1) have an almost periodic mild solution. We will refine the technique of decomposition to further the results obtained above. We first give some lemmas. The following is an easy lemma whose proof is given for the reader's convenience.

Lemma 22. Let $g \in A P(\boldsymbol{X})$ and let $\mathscr{B}$ be an autonomous operator in $B U C(\boldsymbol{R}, \boldsymbol{X})$. Then $\mathscr{B} g \in A P(\boldsymbol{X})$.

Proof. We will use the Bochner criterion for the almost periodicity of a function. Since $g \in A P(\boldsymbol{X})$, we have that the set $\{S(t) g, t \in R\}$ is pre-compact in $B U C(\boldsymbol{R}, \boldsymbol{X})$. On the other hand, as $\mathscr{B}$ is a bounded linear autonomous operator in $B U C(\boldsymbol{R}, \boldsymbol{X})$, we obtain that the set $\{S(t) \mathscr{B} g, t \in R\}=\mathscr{B}\{S(t) g, t \in R\}$ is pre-compact in $B U C(\boldsymbol{R}, \boldsymbol{X})$, that is $\mathscr{B} g \in A P(\boldsymbol{X})$.

Lemma 23. Denote $\boldsymbol{Y}:=B U C(\boldsymbol{R}, \boldsymbol{X})$. For any $g \in \boldsymbol{Y}$, we define the function $G$ as follows:

$$
\begin{aligned}
& G: \boldsymbol{R} \rightarrow \boldsymbol{Y} \\
& G: t \mapsto S(t) g .
\end{aligned}
$$

If $g \in A P(\boldsymbol{X})$, then $G \in A P(\boldsymbol{Y})$ and $\sigma_{b}(G)=\sigma_{b}(g)$.

Proof. Since $g$ is almost periodic, $T(g, \varepsilon)$ is relatively dense in $\boldsymbol{R}$ for all $\varepsilon>0$. On the other hand,

$$
\sup _{t \in \boldsymbol{R}}|G(t+\tau)-G(t)|=\sup _{t \in \boldsymbol{R}}|S(t+\tau) g-S(t) g|=\sup _{s \in \boldsymbol{R}}\|g(\tau+s)-g(s)\|,
$$

where $\|\cdot\|$ and $|\cdot|$ denote the norm in $\boldsymbol{X}$ and $\boldsymbol{Y}$ respectively. We have

$$
T(g, \varepsilon)=T(G, \varepsilon) \quad \forall \varepsilon>0 .
$$

This implies that $T(G, \varepsilon)$ is relatively dense in $\boldsymbol{R}$ for all $\varepsilon>0$, i.e. $G \in A P(\boldsymbol{Y})$. 
Next we prove that $\sigma_{b}(G)=\sigma_{b}(g)$. In fact, we have

$$
\begin{aligned}
a(\lambda, G)(s) & =\lim _{T \rightarrow \infty} \frac{1}{2 T} \int_{-T}^{T} e^{-i \lambda t} S(t) g(s) d t \\
& =\lim _{T \rightarrow \infty} \frac{1}{2 T} \int_{-T}^{T} e^{-i \lambda t} g(t+s) d t \\
& =\lim _{T \rightarrow \infty} \frac{1}{2 T} \int_{-T+s}^{T+s} e^{-i \lambda(\tau-s)} g(\tau) d \tau \\
& =e^{i \lambda s} a(\lambda, g), \quad \forall s \in \boldsymbol{R} .
\end{aligned}
$$

So $a(\lambda, G)=0$ if and only if $a(\lambda, g)=0$, i.e. $\sigma_{b}(G)=\sigma_{b}(g)$.

Definition 24. A function $u \in B U C(\boldsymbol{R}, \boldsymbol{X})$ is said to be a strong mild solution of Eq. (1.1) if there exists a sequence of classical solutions $u_{n}$ to Eq. (1.1) with $f$ replaced by $f_{n} \in B U C(\boldsymbol{R}, \boldsymbol{X})$ such that $\lim _{n \rightarrow \infty} u_{n}=u$, and $\lim _{n \rightarrow \infty} f_{n}=f$ in the sup-norm topology of $B U C(\boldsymbol{R}, \boldsymbol{X})$.

We remark that this concept of strong mild solutions is given in [26] as mild solutions. By the proof of Lemma 16 it is clear that each strong mild solution of Eq. (1.1) is a mild solution of Eq. (1.1). This justifies the terminology we use here. In [24] the reader can find the equivalence of these two concepts of mild solutions when $A$ generates a $C_{0}$-semigroup and $\mathscr{B}=0$.

Lemma 25. Let $u \in A P(\boldsymbol{X})$ be a strong mild solution of Eq. (1.1), $U(t):=S(t) u, F(t):=S(t) f$. Then

$$
\begin{gathered}
a(\lambda, U) \in D(\mathscr{A}), \quad \forall \lambda \in \boldsymbol{R}, \\
a(\lambda, u) \in D(A), \quad \forall \lambda \in \boldsymbol{R},
\end{gathered}
$$

and

$$
(i \lambda-\mathscr{A}-\mathscr{B}) a(\lambda, U)=a(\lambda, F) .
$$

Proof. Since $u$ is a strong mild solution of Eq. (1.1), there exist $\left\{f_{n}\right\} \subset B U C(\boldsymbol{R}, \boldsymbol{X})$ and a sequence of classical solutions $\left\{u_{n}\right\} \subset B U C(\boldsymbol{R}, \boldsymbol{X})$ such that $u_{n} \rightarrow u, f_{n} \rightarrow f$, and

$$
\dot{u}_{n}(t)=A u_{n}(t)+\left[\mathscr{B} u_{n}\right](t)+f_{n}(t) .
$$

Letting $U_{n}(t):=S(t) u_{n}, F_{n}(t):=S(t) f_{n}$, we define the following

$$
a(\lambda, G ; T)(s)=\frac{1}{2 T} \int_{-T}^{T} e^{-i \lambda t} S(t) g(s) d t, \quad \forall \lambda \in \boldsymbol{R}, T>0, s \in \boldsymbol{R}
$$


for all $g \in B U C(\boldsymbol{R}, \boldsymbol{X})$, where $G(t)$ is defined as Lemma 23. Then

$$
\begin{aligned}
a\left(\lambda, S(t) \dot{u}_{n} ; T\right)(s)= & \frac{1}{2 T} \int_{-T}^{T} e^{-i \lambda t} S(t) \dot{u}_{n} d t \\
= & \frac{1}{2 T} \int_{-T}^{T} e^{-i \lambda t} \dot{u}_{n}(t+s) d t \\
= & \frac{1}{2 T} \int_{-T+s}^{T+s} e^{-i \lambda(\tau-s)} \dot{u}_{n}(\tau) d \tau \\
= & i \lambda \frac{1}{2 T} \int_{-T+s}^{T+s} e^{-i \lambda(\tau-s)} u_{n}(\tau) d \tau \\
& +\frac{1}{2 T}\left[u_{n}(T+s) e^{-i \lambda T}-u_{n}(-T+s) e^{i \lambda T}\right] \\
= & i \lambda a\left(\lambda, U_{n} ; T\right)(s)+\frac{1}{2 T}\left[u_{n}(T+s) e^{-i \lambda T}-u_{n}(-T+s) e^{i \lambda T}\right] .
\end{aligned}
$$

So we have

$$
\begin{aligned}
(i \lambda-\mathscr{A}-\mathscr{B}) a\left(\lambda, U_{n} ; T\right)(s)+\frac{1}{2 T}\left[u_{n}(T+s) e^{-i \lambda T}-u_{n}(-T+s) e^{i \lambda T}\right] \\
\quad=a\left(\lambda, F_{n} ; T\right)(s) .
\end{aligned}
$$

Now letting $n \rightarrow \infty$, by the closedness of $(i \lambda-\mathscr{A}-\mathscr{B})$ we obtain

$$
(i \lambda-\mathscr{A}-\mathscr{B}) a(\lambda, U ; T)(s)+\frac{1}{2 T}\left[u(T+s) e^{-i \lambda T}-u(-T+s) e^{i \lambda T}\right]=a(\lambda, F ; T)(s) .
$$

By Lemma 23, $a(\lambda, U)$ and $a(\lambda, F)$ are well defined, and

$$
\lim _{T \rightarrow \infty} a(\lambda, U ; T)=a(\lambda, U), \quad \lim _{T \rightarrow \infty} a(\lambda, F ; T)=a(\lambda, F) .
$$

As $T \rightarrow \infty$, by the closedness of $(i \lambda-\mathscr{A}-\mathscr{B})$ again we obtain (3.11) and (3.13). In the proof of Lemma 23 we proved that $a(\lambda, U)(s)=e^{i \lambda s} a(\lambda, u)$, $\forall s \in \boldsymbol{R}$. Letting $s=0$, we obtain (3.12).

The following theorem is an immediate consequence of Lemmas 23 and 25.

Theorem 26. Let $u \in A P(\boldsymbol{X})$ be a strong mild solution of Eq. (1.1). Then

$$
\sigma_{b}(f) \subset \sigma_{b}(u) \subset \sigma_{i}(\mathscr{A}+\mathscr{B}) \cup \sigma_{b}(f) .
$$

As consequences of Theorem 26 we have. 
Corollary 27. Let $u \in A P(X)$ be a strong mild solution of Eq. (1.1). Then for any finite subset $\Lambda^{1}=\left\{\lambda_{1}, \lambda_{2}, \ldots, \lambda_{N}\right\} \subset \sigma_{i}(\mathscr{A}+\mathscr{B}) \backslash \sigma_{b}(f)$, there exists a mild solution $u_{f} \in A P(X)$, such that

$$
\sigma_{b}\left(u_{f}\right) \subset\left(\sigma_{i}(\mathscr{A}+\mathscr{B}) \backslash \Lambda^{1}\right) \cup \sigma_{b}(f) .
$$

Proof. Let $\quad \Lambda:=\sigma_{i}(\mathscr{A}+\mathscr{B}) \cup \sigma_{b}(f), \quad \Lambda^{2}:=\Lambda \backslash \Lambda^{1}=\left(\sigma_{i}(\mathscr{A}+\mathscr{B}) \backslash \Lambda^{1}\right) \cup$ $\sigma_{b}(f)$, and define

$$
\begin{aligned}
& \Lambda_{A P}:=\left\{g \in A P(\boldsymbol{X}): \sigma_{b}(g) \subset \Lambda\right\}, \\
& \Lambda_{A P}^{1}:=\left\{g \in A P(\boldsymbol{X}): \sigma_{b}(g) \subset \Lambda^{1}\right\}, \\
& \Lambda_{A P}^{2}:=\left\{g \in A P(\boldsymbol{X}): \sigma_{b}(g) \subset \Lambda^{2}\right\} .
\end{aligned}
$$

By the definition of Bohr spectrum, $\Lambda_{A P}, \Lambda_{A P}^{1}, \Lambda_{A P}^{2}$ are closed linear subspaces of $A P(\boldsymbol{X})$ and $\Lambda_{A P}^{1} \cap \Lambda_{A P}^{2}=\{0\}$. So we can easily obtain the decomposition

$$
\Lambda_{A P}=\Lambda_{A P}^{1} \oplus \Lambda_{A P}^{2} \text {. }
$$

Therefore, there exist $u_{1} \in \Lambda_{A P}^{1}, u_{2} \in \Lambda_{A P}^{2}$, where

$$
u_{1}=\sum_{j=1}^{N} a\left(\lambda_{j}, u\right) e^{i \lambda_{j} t},
$$

such that $u=u_{1}+u_{2}$. By Lemma $25, a\left(\lambda_{j}, u\right) \in D(A), j=1,2, \ldots, N$. Letting $f_{1}:=\left(L_{\Lambda}-\mathscr{B}\right) u_{1}, f_{2}:=\left(L_{\Lambda}-\mathscr{B}\right) u_{2}$, we have

$$
\left(L_{\Lambda}-\mathscr{B}\right) u=f_{1}+f_{2}=f .
$$

Since $u_{1}$ is differentiable and $u_{1}(t) \in D(A)$ for all $t$ one may check that $u_{1}$ is a classical solution of Eq. (1.1) with $f$ replaced by $f_{1}$. This yields that $u_{2}$ is a strong mild solutions of Eq. (1.1) with $f$ replaced by $f_{2}$. Now by Theorem 26, we obtain $\sigma_{b}\left(f_{1}\right) \subset \sigma_{b}\left(u_{1}\right) \subset \Lambda^{1}, \sigma_{b}\left(f_{2}\right) \subset \sigma_{b}\left(u_{2}\right) \subset \Lambda^{2}$. This implies that $f_{1} \in \Lambda_{A P}^{1}, f_{2} \in \Lambda_{A P}^{2}$. On the other hand, $f \in \Lambda_{A P}^{2}$, by (3.16) we have $f_{1}=0$ and $f_{2}=f$. Therefore,

$$
\left(L_{\Lambda}-\mathscr{B}\right) u_{2}=f .
$$

This shows that $u_{f}:=u_{2}$ is a mild solution of Eq. (1.1) and (3.15) holds.

Corollary 28. Let $u \in A P(X)$ be a strong mild solution of Eq. (1.1), and let $\sigma_{i}(\mathscr{A}+\mathscr{B})$ be finite. Then there exists a mild solution $u_{f} \in A P(\boldsymbol{X})$, such that $\sigma_{b}\left(u_{f}\right)=\sigma_{b}(f)$.

Proof. It is obtained by Theorem 26 and Corollary 27 immediately.

Consider the case where $f$ is a quasi-periodic function. We have the following result on the non-existence of $k$-quasi-periodic solutions. 
Corollary 29. Let $f$ be a l-quasi periodic function. Then there exists no $k$-quasi periodic strong mild solution of Eq. (1.1) for any $k<l$.

Proof. In fact, if $u$ is $k$-quasi periodic strong mild solution, then by Theorem 26 we have $\sigma_{b}(f) \subset \sigma_{b}(u)$. This implies that $f$ is $k$-quasi periodic. Therefore, this is a contradiction.

Another application of Theorem 26 is illustrated in the following.

Example 30. If $f \in A P(\boldsymbol{X})$ is not $2 \pi$-periodic, that is $\sigma_{b}(f) \not \subset 2 \pi \boldsymbol{Z}$, then Eq. (1.1) has no $2 \pi$-periodic strong mild solutions.

\section{Applications and examples}

Since our operator $\mathscr{B}$ in Eq. (1.1) is very general, our results in the previous section cover many classes of functional differential equations that are considered in the literature. To illustrate this, let $B(\cdot)$ be a function with bounded variation that takes values in $L(\boldsymbol{X})$, where $L(\boldsymbol{X})$ denotes the space of all bounded linear operators from $\boldsymbol{X}$ to $\boldsymbol{X}$, and let

$$
\int_{-\infty}^{\infty}\|d B(\eta)\| e^{\delta|\eta|}<\infty
$$

for some $\delta>0$. Then we can define

$$
[\mathscr{B} u](t)=\int_{-\infty}^{\infty} d B(\eta) u(t+\eta), \quad \forall u \in B U C(\boldsymbol{R}, \boldsymbol{X}), t \in \boldsymbol{R} .
$$

Consider equations of the form

$$
\dot{u}(t)=A u(t)+\int_{-\infty}^{\infty} d B(\eta) u(t+\eta)+f(t), \quad t \in \boldsymbol{R} .
$$

Let $u \in B U C(\boldsymbol{R}, \boldsymbol{X})$ be a mild solution of (4.2). By the same method as in the proof of Lemma 14 we can show that $\mathscr{D}-\mathscr{A}-\mathscr{B}$ is closable in $B U C(\boldsymbol{R}, \boldsymbol{X})$. Moreover, because of the closedness of the operator $L$ with domain consisting of all $u \in B U C(\boldsymbol{R}, \boldsymbol{X})$ such that $\int_{s}^{t} u(\xi) d \xi \in D(A)$ for all $t \geq s$, and

$$
u(t)-u(s)=A \int_{s}^{t} u(\xi) d \xi+\int_{s}^{t} g(\xi) d \xi
$$

for some $g \in B U C(\boldsymbol{R}, \boldsymbol{X})$, and $L u=g$, the operator $L-\mathscr{B}$ is a closed extension of $\mathscr{D}-\mathscr{A}-\mathscr{B}$. This implies that each mild solution in the sense of Definition 11 satisfies the equation

$$
u(t)-u(s)=A \int_{s}^{t} u(\xi) d \xi+\int_{s}^{t} \int_{-\infty}^{\infty} d B(\eta) u(\eta+\xi) d \xi+\int_{s}^{t} f(\xi) d \xi, \quad t \geq s .
$$


In this case we will show that the set $\sigma_{i}(\mathscr{A}+\mathscr{B})$ can be replaced by a simpler one that can be easier computed. In the proof of Theorem 15 we got the following equation

$$
(\lambda-\mathscr{D})^{-1} f+u=[\lambda-(\mathscr{A}+\mathscr{B})](\lambda-\mathscr{D})^{-1} u, \quad \operatorname{Re} \lambda \neq 0 .
$$

Recall that

$$
(\lambda-\mathscr{D})^{-1} u(\xi)= \begin{cases}\int_{0}^{\infty} e^{-\lambda t} u(t+\xi) d \xi, & (\text { if } \operatorname{Re} \lambda>0) \\ -\int_{-\infty}^{0} e^{-\lambda t} f(t+\xi) d \xi, & (\text { if } \operatorname{Re} \lambda<0)\end{cases}
$$

and

$$
\hat{u}(\lambda)=(\lambda-\mathscr{D})^{-1} u(0)
$$

We have

$$
\begin{aligned}
(\lambda-\mathscr{D})^{-1} f(0)+u(0) & =[\lambda-(\mathscr{A}+\mathscr{B})](\lambda-\mathscr{D})^{-1} u(0) \\
& =\left[\lambda(\lambda-\mathscr{D})^{-1} u-\mathscr{A}(\lambda-\mathscr{D})^{-1} u-(\lambda-\mathscr{D})^{-1} \mathscr{B} u\right](0) \\
& =\lambda \hat{u}(\lambda)-A \hat{u}(\lambda)-\left[(\lambda-\mathscr{D})^{-1} \mathscr{B} u\right](0) .
\end{aligned}
$$

In the case of $\delta>\operatorname{Re} \lambda>0$ we have

$$
\begin{aligned}
{\left[(\lambda-\mathscr{D})^{-1} \mathscr{B} u\right](0) } & =\int_{0}^{\infty} e^{-\lambda t}[\mathscr{B} u](t) d t \\
& =\int_{0}^{\infty} e^{-\lambda t} \int_{-\infty}^{\infty} d B(\eta) u(\eta+t) d t \\
& =\int_{-\infty}^{\infty} d B(\eta) \int_{0}^{\infty} e^{-\lambda t} u(\eta+t) d t \\
& =\int_{-\infty}^{\infty} d B(\eta) e^{-\lambda \eta}\left(\int_{0}^{\infty} e^{-\lambda \xi} u(\xi) d \xi-\int_{0}^{\eta} e^{-\lambda \xi} u(\xi) d \xi\right) \\
& =\int_{-\infty}^{\infty} d B(\eta) e^{-\lambda \eta} \hat{u}(\lambda)-\int_{-\infty}^{\infty} d B(\eta) e^{-\lambda \eta} \int_{0}^{\eta} e^{-\lambda \xi} u(\xi) d \xi
\end{aligned}
$$

In the case of $-\delta<\operatorname{Re} \lambda<0$, we obtain a similar equality. So we have

$\hat{f}(\lambda)+u(0)=\lambda \hat{u}(\lambda)-A \hat{u}(\lambda)-\int_{-\infty}^{\infty} d B(\eta) e^{-\lambda \eta} \hat{u}(\lambda)+\int_{-\infty}^{\infty} d B(\eta) e^{-\lambda \eta} \int_{0}^{\eta} e^{-\lambda \xi} u(\xi) d \xi$

that is,

$$
\hat{f}(\lambda)+u(0)-\int_{-\infty}^{\infty} d B(\eta) e^{-\lambda \eta} \int_{0}^{\eta} e^{-\lambda \xi} u(\xi) d \xi=\left(\lambda-A-\int_{-\infty}^{\infty} d B(\eta) e^{-\lambda \eta}\right) \hat{u}(\lambda) .
$$


If $\xi \notin \Sigma$, where

$$
\Sigma:=\left\{z \in \boldsymbol{C}:|\operatorname{Re} z|<\delta, \nexists\left(z-A-\int_{-\infty}^{\infty} d B(\eta) e^{-z \eta}\right)^{-1} \in L(\boldsymbol{X})\right\},
$$

we have

$$
\hat{u}(\lambda)=\left(\lambda-A-\int_{-\infty}^{\infty} d B(\eta) e^{-\lambda \eta}\right)^{-1}\left(\hat{f}(\lambda)+u(0)-\int_{-\infty}^{\infty} d B(\eta) e^{-\lambda \eta} \int_{0}^{\eta} e^{-\lambda \xi} u(\xi) d \xi\right) .
$$

We can prove (see e.g. [8]) that if $i \xi \notin \Sigma$ and $\xi \notin s p(f)$, then

$$
\left(\lambda-A-\int_{-\infty}^{\infty} d B(\eta) e^{-\lambda \eta}\right)^{-1}, \quad \int_{-\infty}^{\infty} d B(\eta) e^{-\lambda \eta} \int_{0}^{\eta} e^{-\lambda \xi} u(\xi) d \xi
$$

are analytic in $\lambda$ around $i \xi$. This implies $\xi \notin s p(u)$, so $s p(u) \subset \Sigma_{i} \cup s p(f)$, where $\Sigma_{i}:=\{\xi \in \Sigma: i \xi \in \Sigma\}$. So we arrive at

Proposition 31. Let the above mentioned conditions for Eq. (4.2) be satisfied, and let $u \in B U C(\boldsymbol{R}, \boldsymbol{X})$ be a mild solution of Eq. (4.2). Then,

$$
s p(u) \subset \Sigma_{i} \cup s p(f) .
$$

The advantage of the estimate (4.5) is that we need only to study the "characteristic roots" of the equation, instead of the spectrum $\sigma_{i}(\mathscr{A}+\mathscr{B})$. We are ready to see how our results obtained above extend respective ones in [8]. Now the reader can re-state all above conditions and results in terms of the spectral set $\Sigma_{i}$, instead of $\sigma_{i}(\mathscr{A}+\mathscr{B})$.

\section{Acknowledgement}

The third author N.V.M. thanks the Beijing Normal University for the support during his visit in summer 2006. The authors of the paper are grateful to anonymous referees and the editor for pointing out several inaccuracies and for the suggestions to improve the presentation of the paper.

\section{References}

[ 1 ] Arendt, W. and Batty, C. J. K., Almost periodic solutions of first and second oder Cauchy problems, J. Differential Equations, 137 (1997), 363-383.

[2 ] Arendt, W., Batty, C. J. K., Hieber, M. and Neubrander, F., Vector-valued Laplace transforms and Cauchy problems, Monographs in Mathematics, 96, Birkhäuser, Basel, 2001.

[3] Benkhalti, R. and Ezzinbi, K., A Massera type criterion for some partial functional differential equations, Dynam. Systems Appl., 9 (2000), 221-228.

[4] Chow, S. N. and Hale, J. K., Strongly limit-compact maps, Funkcial. Ekva, 17 (1974), 3138 . 
[ 5 ] Diagana, T., N'guérekata, G. and Minh, N. V., Almost automorphic solutions of evolution equations, Proc. Amer. Math. Soc., 132 (2004), 3289-3298.

[6] Engel, K. J. and Nagel, R., One-parameter Semigroups for linear Evolution Equations, Springer, Berlin, 1999.

[ 7] Ezzinbi, K. and Jazar, M., New criteria for the existence of periodic and almost periodic solutions for some evolution equations in Banach spaces, Electron. J. Qual. Theory Differ. Equ., (2004), No. 6, 12 pp. (electronic).

[ 8 ] Furumochi, T., Naito, T. and Minh, N. V., Boundedness and almost periodicity of solutions of partial functional differential equations, J. Differential Equations, 180 (2002), 125-152.

[9] Hale, J., Theory of Functional Differential Equations, Acad. Press, New York, 1977.

[10] Henry, D., Geometric Theory of Semilinear Parabolic Equations, Lecture Notes in Math., Springer-Verlag, Berlin-New York, 1981.

[11] Hernández, M. E., A Massera type criterion for a partial neutral functional differential equation, Electron. J. Differential Equations, 2002 (2002), No. 40, 17 pp.

[12] Hino, Y., Naito, T., Minh, N. V. and Shin, J. S., Almost Periodic Solutions of Differential Equations in Banach Spaces, Taylor \& Francis, London-New York, 2002.

[13] Kato, T., Perturbation Theory for Linear Operators (Second edition), Grundlehren der Mathematischen Wissenschaften, Band 132. Springer-Verlag, Berlin-New York, 1976.

[14] Levitan, B. M. and Zhikov, V. V., Almost Periodic Functions and Differential Equations, Moscow Univ. Publ. House 1978, English translation by Cambridge University Press 1982.

[15] Li, Y., Lin, Z. and Li, Z., A Massera type criterion for linear functional differential equations with advanced and delay, J. Math. Anal. Appl., 200 (1996), 715-725.

[16] Li, Y., Cong, F., Lin, Z. and Liu, W., Periodic solutions for evolution equations, Nonlinear Anal. Ser. A: Theory Methods, 36 (1999), 275-293.

[17] Liu, J., Nguerekata, G., Minh, N. V. and Phong, V. Q., Bounded solutions of parabolic equations in continuous function spaces, To appear in Funkcial. Ekva.

[18] Massera, J. L., The existence of periodic solutions of systems of differential equations, Duke Math. J., 17 (1950), 457-475.

[19] Minh, N. V. and Minh, H. B., A Massera-type criterion for almost periodic solutions of higher-order delay or advance abstract functional differential equations, Abstr. Appl. Anal., (2004), 881-896.

[20] Murakami, S., Naito, T. and Minh, N. V., Evolution semigroups and sums of commuting operators: A new approach to the admissibility theory of function spaces, J. Differential Equations, 164 (2000), 240-285.

[21] Murakami, S., Naito, T. and Minh, N. V., Massera's theorem for almost periodic solutions of functional differential equations, J. Math. Soc. Japan, 56 (2004), 247-268.

[22] Naito T. and Minh, N. V., Evolutions semigroups and spectral criteria for almost periodic solutions of periodic evolution equations, J. Differential Equations, 152 (1999), 358-376.

[23] Naito, T., Minh, N. V. and Shin, J. S., New spectral criteria for almost periodic solutions of evolution equations, Studia Math., 145 (2001), 97-111.

[24] Pazy, A., Semigroups of Linear Operators and Applications to Partial Differential Equations, Applied Math. Sci., 44, Spriger-Verlag, Berlin-New York 1983.

[25] Prüss, J., Bounded solutions of Volterra equations, SIAM J. Math. Anal., 19 (1987), 133-149.

[26] Prüss, J., Evolutionary Integral Equations and Applications, Birkhäuser, Basel, 1993.

[27] Shin, J. S. and Naito, T., Semi-Fredholm operators and periodic solutions for linear functional differential equations, J. Differential Equations, 153 (1999), 407-441.

[28] Zaidman, S., On the Bohr transform of almost-periodic solutions for some differential equations in abstract spaces, Int. J. Math. Math. Sci., 27 (2001), 521-534. 


\author{
nuna adreso: \\ Qing Liu \\ Laboratory of Mathematics and Complex \\ Systems \\ School of Mathematical Science \\ Beijing Normal University \\ Beijing 100875 \\ P.R. China \\ E-mail: bnumlq@mail.bnu.edu.cn \\ Nguyen Van Minh \\ Department of Mathematics \\ University of West Georgia \\ Carrollton, GA 30118 \\ USA \\ E-mail: vnguyen@westga.edu \\ G. Nguerekata \\ Department of Mathematics \\ Morgan State University \\ 1700E. Cold Spring Lane \\ Baltimore, MD21251 \\ USA \\ E-mail: gnguerek@jewel.morgan.edu \\ Rong Yuan \\ Laboratory of Mathematics and Complex \\ Systems \\ School of Mathematical Science \\ Beijing Normal University \\ Beijing 100875 \\ P.R. China \\ E-mail: ryuan@bnu.edu.cn
}

(Ricevita la 15-an de marto, 2007)

(Reviziita la 14-an de septembro, 2007) 